\title{
Renal Sarcoidosis and Multiple Myeloma
}

\author{
C Gashti, A Vijaykumar, M Larson
}

\author{
Citation \\ C Gashti, A Vijaykumar, M Larson. Renal Sarcoidosis and Multiple Myeloma. The Internet Journal of Nephrology. 2009 \\ Volume 6 Number 1.
}

DOI: $\underline{10.5580 / 12 \mathrm{e} 1}$

\begin{abstract}
An association between sarcoidosis and multiple myeloma (MM) has been reported in several case reports. In these cases, sarcoidosis is chronically active, systemic, diagnosed at a later age than controlled groups, and often precedes the diagnosis of multiple myeloma by several years. We present the first case of a 43-year-old asymptomatic man with abnormal renal function and monoclonal gammopathy who was simultaneously diagnosed with renal sarcoidosis and MM. He was successfully treated with Thalidomide and steroids and remains in remission after two years of follow up. Pathophysiology of sarcoidosis and MM with special focus on potential link between the two diseases is discussed.
\end{abstract}

\section{INTRODUCTION}

Sarcoidosis is a chronic inflammatory disease with clinically significant renal involvement mostly due to hypercalcemia (10\%) and hypercalciuria (50\%). ${ }^{1}$ Up to $20 \%$ of sarcoidosis patients show granulomatous interstitial nephritis on biopsy and autopsy reports. ${ }^{2}$ An association between sarcoidosis and malignancy has been suggested. ${ }^{3}$ Hodgkin's disease and nonHodgkin lymphoma encompass the majority of cases that highlight this association.

Less commonly, monoclonal gammopathies have been reported in association with sarcoidosis. There are eleven case reports of multiple myeloma (MM) in association with sarcoidosis in the literature. ${ }^{4}$ None of these cases report renal involvement. We describe a patient with simultaneous presentation of renal sarcoidosis and MM. We discuss the atypical presentation, pathophysiology and highlight the importance of performing a renal biopsy.

\section{CASE REPORT}

A 43-year-old African-American male with no prior medical history was referred to outpatient nephrology clinic for evaluation of abnormal renal function found on routine health exam. Serum creatinine level was $2.4 \mathrm{mg} / \mathrm{dL}$. Review of systems were unremarkable. He was not on any medications. His blood pressure was 138/104 mmHg. Physical examination findings were unremarkable. A complete blood count showed a white blood cell count of 4.5 x $10^{9} \mathrm{~L}$, hemoglobin $13.3 \mathrm{~g} / \mathrm{dL}$ and platelet count of $135 \mathrm{x}$
$10^{9} \mathrm{~L}$. Serum calcium was $9.5 \mathrm{mg} / \mathrm{dL}$ with an albumin of 3.9 $\mathrm{g} / \mathrm{dL}$ and a total protein level of $9.7 \mathrm{~g} / \mathrm{dL}$. Urinalysis showed trace blood and 1+ protein with no cellular casts. He had 480 $\mathrm{mg}$ of proteinuria on a 24 hour urine collection. Urine calcium to creatinine ratio was within normal range (121 $\mathrm{mg} / \mathrm{g}$ ). Both serum and urine protein electrophoresis with immunofixation revealed monoclonal Immunoglobulin (Ig) $\mathrm{G}$ lambda. Quantitative serum Igs showed $43 \mathrm{~g} / \mathrm{L}$ (normal range, 6-16 g/L) of IgG and normal levels of $\mathrm{IgA}$ and $\mathrm{IgM}$. Lambda free light chains were elevated at $96.3 \mathrm{mg} / \mathrm{L}$ (normal range, 5.17-26.3 mg/L) with normal kappa free light chains of $17.8 \mathrm{mg} / \mathrm{L}$ (normal range, 3.3-19.4 mg/L). Skeletal survey radiography was negative for osteolytic bone lesions.

Bone marrow biopsy and aspiration was consistent with MM without any evidence of granulomas. Percutaneous renal biopsy showed areas of non-caseating granulomatous interstitial nephritis involving approximately $50 \%$ of the cortex and medulla (Figure 1). The uninvolved areas of cortex contained 17 glomeruli and were unremarkable by light and immunofluorescent microscopy. The tubules were free of casts (Figure 2). Stains for acid fast (Fite's) and fungal (GMS) were negative. The congo red stain in both the bone marrow and renal tissues were negative. Infectious workup including urine and sputum cultures for acid fast bacilli was negative. Serum Angiotensin Converting Enzyme (ACE) level was elevated at $81 \mathrm{U} / \mathrm{L}$ with a normal 1,25dihydroxy Vitamin D level of $23 \mathrm{pg} / \mathrm{ml}$. Computed Tomography scan of the chest, abdomen and pelvis revealed 
hilar and para-aortic abdominal lymphadenopathy consistent with the diagnosis of sarcoidosis.

The patient was started on prednisone at $1 \mathrm{mg} / \mathrm{kg}$ for treatment of renal sarcoidosis. He was subsequently switched to Thalidomide and Dexamethasone for treatment of multiple myeloma and remained on this therapy for 5 months. After two years of follow up, his serum creatinine was $2.15 \mathrm{mg} / \mathrm{dL}$. Quantitative serum IgG had decreased to $21 \mathrm{~g} / \mathrm{L}$. He remains asymptomatic without any signs of anemia or hypercalcemia.

\section{Figure 1}

Fig 1. Granulomatous interstitial nephritis. Ischemic glomerulus surrounded by isolated atrophic tubules and granulomatous inflammation with sheets of epithelioid histiocytes. On the right side of the picture is a well-formed granuloma. PAS x132.

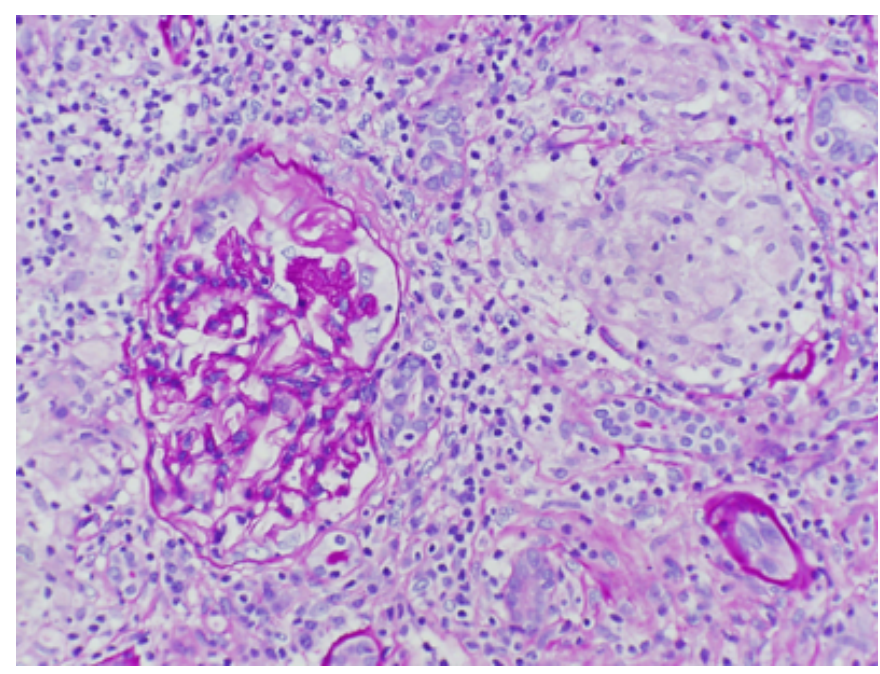

\section{Figure 2}

Fig 2. Granulomatous interstitial nephritis. Renal tubule surrounded by granulomatous inflammation with sheets of epithelioid histiocytes. The tubule shows tubulitis with lymphocytes invading the tubular epithelium. PAS x132.

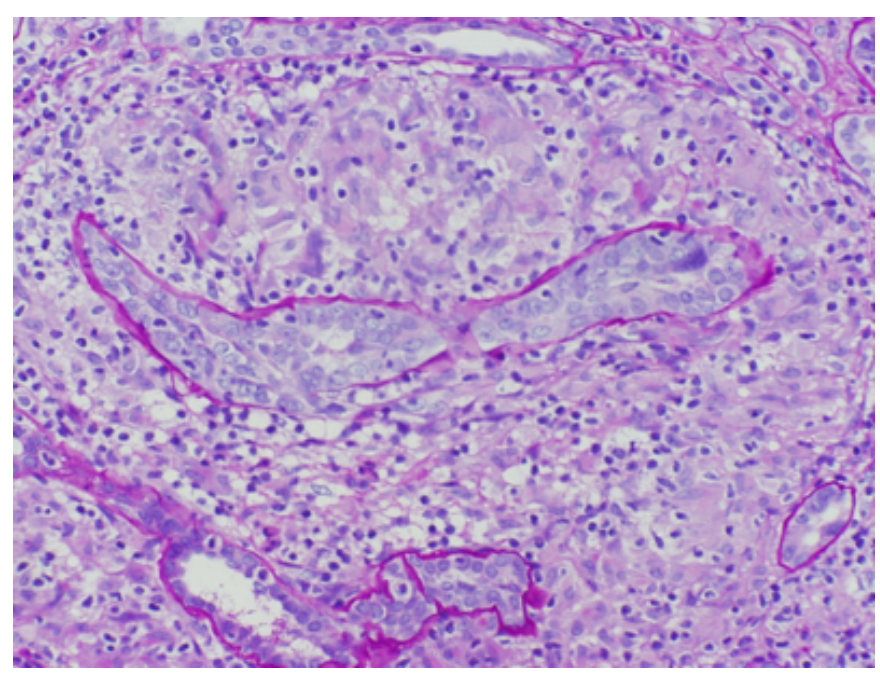

\section{DISCUSSION}

MM is a lymphoid neoplasm resulting from genetic changes in late B-cell differentiation. ${ }^{5}$ The incidence of $\mathrm{MM}$ is estimated at 4.2 per 100,000 population and patients $40-49$ years of age comprise $8 \%$ of these cases. ${ }^{6}$ The etiology of $\mathrm{MM}$ is unknown, however, in addition to environmental exposures, chronic inflammatory diseases leading to prolonged stimulation of the reticuloendothelial system in animal models have been implicated. ${ }^{7}$

Sarcoidosis, a multiorgan granulomatous inflammatory disease, has been linked to increased activity of macrophages and $\mathrm{CD} 4^{+}$Th cells. ${ }^{8}$ In addition to infectious causes such as mycobacterial and propionibacterial organisms, mutations of butyrophilin-like 2 (BTNL2), an MHC class II gene-linked butyrophilin-like molecule have been linked to sarcoidosis. ${ }^{9 ; 10}$ The annual incidence of sarcoidosis is 35 per 100,000 in the African-American population. ${ }^{11}$ Based on these figures, the coincidental occurrence of MM and sarcoidosis in the same patient is rare and may suggest a causal relationship.

Two competing theories have attempted to explain the observed association between MM and sarcoidosis. ${ }^{4}$ The original hypothesis suggests that active chronic inflammation from sarcoidosis is responsible for chronic activation of $\mathrm{CD}^{+}$Th cells and decreased $\mathrm{CD} 8^{+} \mathrm{T}$ suppressor cells, which result in continuous stimulation of B-cells and subsequent malignant transformation. The counter hypothesis using linkage-analysis proposes a 
systemic sarcoid-like reaction to malignancy. In nine of the twelve reported cases, sarcoidosis preceded the diagnosis of MM, which supports the original hypothesis of sarcoidosis as a potential cause for malignancy. The B7 superfamily of receptors that are upregulated in antigen-presenting cells, such as macrophages, have been shown to play a crucial role in sarcoidosis. ${ }^{12}$ The interaction between $\mathrm{B} 7$ receptors and CD28 on naïve T-cells is a co-stimulatory signal for cell activation and tolerance. ${ }^{13}$ However, CD28 is present on some malignant plasma cells and its expression in MM patients is associated with disease progression and treatment failure, a potential common link between the two diseases. ${ }^{14}$

The common themes in the eleven previously reported cases of sarcoidosis and MM are chronic active sarcoidosis, diagnosis of MM an average of 6 years (range 2-27 years) after the diagnosis of sarcoidosis, and older age of patients (56 years) at the time sarcoidosis was diagnosed as compared to unselected sarcoidosis patients. ${ }^{4}$ The current case has an atypical presentation that challenges these previously observed features. Our patient presented at a younger age of 43, not atypical for sarcoidosis but rather rare for MM. In addition, to our knowledge, this is the only case of MM and renal sarcoidosis. There was no evidence of paraproteinemia-associated renal lesion or granulomatous involvement of the bone marrow. The diagnosis of sarcoidosis and MM were made simultaneously, although the patient likely had undiagnosed renal sarcoid for an unknown period of time given the degree of chronic interstitial fibrosis.

This case highlights the importance of performing a renal biopsy in the setting of paraproteinemia. Paueksakon et al. studied the renal biopsies of 87 patients with monoclonal gammopathy and found $63 \%$ of this group had renal disease unrelated to the paraproteinemia. ${ }^{15}$ With the bone marrow findings of MM, if assumed that the renal insufficiency was paraprotein-related, the diagnosis of sarcoidosis would have been missed.

Recently, the French Sarcoidosis Group reported 47 cases of biopsy-proven renal sarcoidosis. Renal sarcoidosis has a high prevalence of hypercalcemia, which was not a feature of our patient. More importantly, the predictors of long-term renal outcome in steroid-treated renal sarcoidosis patients are directly related to response at 1 month and inversely related to the degree of interstitial fibrosis. ${ }^{16}$ In our patient, despite steroid therapy, serum creatinine continued to rise for the first 3 months of therapy before it stabilized and subsequently returned to pre-treatment values. This incomplete renal response seen at one month and two-year follow up is explained by the significant interstitial fibrosis $(>50 \%)$ on renal biopsy.

\section{ACKNOWLEDGEMENT}

We thank Dr Melvin Schwartz (Pathology, Rush University Medical Center) for supplying the histological images.

\section{References}

1. Gobel U, Kettritz R, Schneider W, Luft F. The protean face of renal sarcoidosis. J Am Soc Nephrol 2001; 12: 616-623

2. LONGCOPE WT, FREIMAN DG. A study of sarcoidosis; based on a combined investigation of 160 cases including 30 autopsies from The Johns Hopkins Hospital and Massachusetts General Hospital. Medicine (Baltimore) 1952; 31: 1-132

3. Brincker H. The sarcoidosis-lymphoma syndrome. Br J Cancer 1986; 54: 467-473

4. Sen F, Mann KP, Medeiros LJ. Multiple myeloma in association with sarcoidosis. Arch Pathol Lab Med 2002; 126: $365-368$

5. Billadeau D, Ahmann G, Greipp P, Van NB. The bone marrow of multiple myeloma patients contains B cell populations at different stages of differentiation that are clonally related to the malignant plasma cell. J Exp Med 1993; 178: 1023-1031

6. Kyle RA, Gertz MA, Witzig TE et al. Review of 1027 patients with newly diagnosed multiple myeloma. Mayo Clin Proc 2003; 78: 21-33

7. Wiernick P, Canellos G, Dutcher J, Kyle R. Neoplastic Diseases of the Blood. Churchill Livingstone, New York: 1996; 423

8. Newman LS, Rose CS, Maier LA. Sarcoidosis. N Engl J Med 1997; 336: 1224-1234

9. Chen ES, Moller DR. Etiology of sarcoidosis. Clin Chest Med 2008; 29: 365-77, vii

10. Rybicki BA, Walewski JL, Maliarik MJ, Kian H, Iannuzzi MC. The BTNL2 gene and sarcoidosis susceptibility in African Americans and Whites. Am J Hum Genet 2005; 77: 491-499

11. Rybicki BA, Major M, Popovich J, Jr., Maliarik MJ, Iannuzzi MC. Racial differences in sarcoidosis incidence: a 5 -year study in a health maintenance organization. Am J Epidemiol 1997; 145: 234-241

12. Nguyen T, Liu XK, Zhang Y, Dong C. BTNL2, a butyrophilin-like molecule that functions to inhibit $\mathrm{T}$ cell activation. J Immunol 2006; 176: 7354-7360

13. Janeway CA, Jr., Medzhitov R. Innate immune recognition. Annu Rev Immunol 2002; 20: 197-216 14. Robillard N, Jego G, Pellat-Deceunynck C et al. CD28, a marker associated with tumoral expansion in multiple myeloma. Clin Cancer Res 1998; 4: 1521-1526

15. Paueksakon P, Revelo MP, Horn RG, Shappell S, Fogo

AB. Monoclonal gammopathy: significance and possible causality in renal disease. Am J Kidney Dis 2003; 42: 87-95

16. Mahevas M, Lescure FX, Boffa JJ et al. Renal sarcoidosis: clinical, laboratory, and histologic presentation and outcome in 47 patients. Medicine (Baltimore) 2009; 88: 98-106 


\section{Author Information}

Casey N. Gashti, MD

Department of Nephrology, Rush University Medical Center

\section{Aarthi Vijaykumar, MD}

Department of Nephrology, Rush University Medical Center

\section{Melissa L. Larson, MD}

Department of Hematology and Oncology, Rush University Medical Center 